\title{
FACTORES PSICOSOCIALES PREDICTORES DE LA CALIDAD DE VIDA EN PERSONAS EN SITUACIÓN DE DISCAPACIDAD FÍSICA
}

\author{
PAULA ANDREA BOTERO SOTO \\ CORPORACIÓN ECCOS CONTACTO COLOMBIA \\ CONSTANZA LONDOÑO PÉREZ* \\ UNIVERSIDAD CATÓLICA DE COLOMBIA
}

Recibido, octubre 14/2012

Concepto evaluación, mayo 17/2013

Aceptado, julio 20/2013
Referencia: Botero, P. A \& Londoño, C. (2013). Factores psicosociales predictores de la calidad de vida en personas en situación de discapacidad física. Acta Colombiana de Psicología, 16 (2), 125-137.

Resumen

El presente estudio correlacional descriptivo se propuso evaluar los factores que determinan la calidad de vida (CV) relacionada con la salud en 78 personas con discapacidad física que vivían en Bogotá, y hacían parte de un proyecto de inclusión laboral dirigido por una ONG. Se evaluó la calidad de vida relacionada con la salud (Cuestionario de Salud SF36 y visita domiciliaria), al igual que las condiciones asociadas a la discapacidad y algunos factores psicológicos (Escala de Autoeficacia Generalizada, Prueba de Imagen Corporal, Inventario de Estilos de Afrontamiento, Prueba de optimismo disposicional, Inventario de Depresión de Beck y Escala de Ansiedad de Zung). Se utilizó un modelo de ecuaciones estructurales para encontrar el poder predictivo de las variables en la calidad de vida. El modelo obtenido predecía un 39\% de la varianza, incluyendo en orden de peso relativo aportando variables como condiciones de enfermedad, depresión, afrontamiento centrado en la fantasía, trascendencia, pesimismo, ansiedad y autoeficacia. Se discuten los hallazgos y las limitaciones del estudio.

Palabras clave: calidad de vida, discapacidad física, factores psicológicos

\section{PSYCHOSOCIAL PREDICTORS OF QUALITY OF LIFE IN PEOPLE EXPERIENCING PHYSICAL DISABILITY}

\begin{abstract}
This descriptive correlational study aimed to assess the factors that determine the quality of life related to health in 78 people with physical disabilities who lived in Bogotá and were part of a labor inclusion project run by a NGO. Quality of life related to health was assessed with the SF36 Health Questionnaire and a home visit, as well as the conditions associated with disability and some psychological factors which were measured with the Generalized Self-Efficacy Scale, Body Image Test, Coping Styles Inventory, Dispositional Optimism Test, Beck Depression Inventory and Zung Anxiety Scale. A structural equation model was used in order to determine the predictive power of the quality of life variables. The obtained model predicted $39 \%$ of the variance, including the variables' relative weight order, and provided variables as disease condition, depression, coping style focused on fantasy, transcendence, pessimism, anxiety and self-efficacy. Findings and limitations of the study are discussed.

Key Words: Quality of life, physical disability, psychological factors.
\end{abstract}

\section{FATORES PSICOSSOCIAIS PRECEPTORES DA QUALIDADE DE VIDA NAS PESSOAS EM COM NECESSIDADES ESPECIAIS'}

Resumo

\begin{abstract}
O presente estudo correlacional descritivo se propôs avaliar os fatores que determinam a qualidade de vida relacionada com a saúde em 78 pessoas com necessidades especiais que moravam em Bogotá, e faziam parte de um projeto de inclusão trabalhista dirigido por uma ONG. Avaliou-se a qualidade de vida relacionada com a saúde (Questionário de Saúde SF36 e visita domiciliar), da mesma forma que as condições associadas à necessidade especial e alguns fatores psicológicos (Escala de Autoeficácia Generalizada, Teste de Imagem Corporal, Inventário de Estilos de Enfrentamento, Teste de otimismo disposicional, Inventário de Depressão de Beck e Escala de Ansiedade de Zung). Utilizou-se um modelo de equações estruturais para encontrar o poder preditivo das variáveis na qualidade de vida. O modelo obtido predizia 39\% de variância, incluindo em ordem de peso relativo contribuindo variáveis como condições de doença, depressão, enfrentamento centrado na fantasia, transcendência, pessimismo, ansiedade e autoeficácia. Discutem-se as descobertas e as limitações do estudo.

Palavras chave: qualidade de vida, incapacidade de vida, deficiência física, fatores psicológicos.
\end{abstract}

clondono@ucatolica.edu.co 
En la actualidad, aproximadamente 600 millones de personas en el mundo tienen discapacidades de diversos tipos, la mayoría provenientes de países en vía de desarrollo (Organización Mundial de la Salud [OMS], 2005), con prevalencia en Centroamérica y Suramérica con un 14.5\% (Banco Interamericano de Desarrollo, 2007). En Colombia, se ha dado un incremento sustancial de la población en situación de discapacidad que asciende a 2'651.701 personas (DANE, 2005).

La Organización Mundial de la Salud OMS (2001) ha formulado en la Clasificación Internacional del Funcionamiento, la Discapacidad y la Salud (CIF) un modelo integrador biopsicosocial para conceptualizar y categorizar la discapacidad. Desde esta perspectiva se entiende la discapacidad como las deficiencias en las funciones y/o estructuras corporales, las limitaciones en la actividad y las restricciones en la participación que presenta una persona que tiene una condición de salud en interacción con factores ambientales y personales.

La discapacidad puede ser conceptualizada como una forma de funcionamiento de la persona en relación con las capacidades que tiene, las estructuras corporales y las expectativas de su entorno personal y social. Esta situación afecta de distintos modos la calidad de vida de las personas que la viven, dependiendo de la severidad de la misma. En general, se observa que la investigación en el tema de calidad de vida en personas con discapacidad ha avanzado sustancialmente, afinando cada vez más, tanto la definición de la que se parte, como la forma de medición del constructo, de tal modo que sirve como un indicador de éxito de los programas e iniciativas políticas sobre las acciones de prevención e intervención con discapacidad (Córdoba, Gómez, \& Verdugo, 2008; Velasco, Botero, \& Londoño, 2011).

La Calidad de Vida Relacionada con la Salud (CVRS) puede definirse como la percepción subjetiva de las personas sobre la capacidad para realizar actividades importantes en la vida, influenciada por el estado de salud actual. Esta valoración subjetiva del bienestar es determinada por la percepción de las limitaciones físicas, psicológicas, sociales y de disminución de oportunidades como consecuencia de una enfermedad, secuelas de la misma, tratamientos y servicios médicos de acceso (Schwartzmann, 2003). La CVRS, desde una dimensión subjetiva, implica la evaluación en relación con el estado emocional, físico y social que presenta en relación con la satisfacción con los aspectos fisiológicos (síntomas, discapacidad funcional, sueño, respuesta sexual); emocionales (tristeza, miedo, inseguridad, frustración), y sociales (situación laboral, académica, interacción social, familia, nivel económico, actividades comunitarias y de ocio) (Vinaccia et al., 2006a).
En general, las investigaciones han abordado el tema de la calidad de vida global y la calidad de vida relacionada con la salud en pacientes de diversas partes del mundo que presentan enfermedades o condiciones de salud crónicas. Tal es el caso de las investigaciones realizadas en pacientes con epilepsia (Endermann \& Zimmermann, 2009), diversos tipos de cáncer (Gaviria et al., 2007; Costa Neto, \& Cavalcanti, 2005); enfermedad pulmonar obstructiva crónica EPOC (Taylor et al., 2008; Vinaccia et al., 2006b; Pérez et al., 2005); insuficiencia renal crónica (Contreras et al., 2007; Pucheu, et al., 2004); pacientes con fallas cardiacas y enfermedades cardiovasculares crónicas (Krethong et al., 2007); hiperplasia prostática (Vinaccia et al., 2006a); dispepsia no ulcerosa (Cano et al., 2006); fibrosis quística (Abbott et al., 2008); diabetes mellitus tipo 2 (Pirkola et al., 2009), y pacientes con dolor crónico (Taylor, Dean, \& Siegert, 2006). En el campo de la salud mental se ha estudiado la calidad de vida y la calidad de vida relacionada con la salud en pacientes diagnosticados con esquizofrenia (Awad, Voruganti, \& Heslegrave, 1997; Caqueo \& Lemos, 2008; Narváez et al., 2008); desorden obsesivo compulsivo (Albert et al., 2009), y diagnóstico de depresión (Gallegos et al., 2009).

Específicamente, en el campo de la discapacidad se han realizado estudios que abordan condiciones de discapacidad en general (Uppal, 2006) o discapacidad física o psiquiátrica (Anctil, et al., 2007). También se encuentran otro tipo de estudios que abordan condiciones de salud específicas que generan limitaciones en la funcionalidad física, tales como lupus eritematoso sistémico (Vinaccia et al., 2006b), artritis reumatoide (Kojima et al., 2009), secuelas de postpolio (Stuifbergen et al., 2005) y discapacidades específicas como amputaciones de miembro superior o inferior por causas adquiridas (Desmond \& MacLachlan, 2006). Adicionalmente, se han hecho trabajos orientados hacia la calidad de vida de familias que tienen hijos con discapacidad, teniendo en cuenta que las limitaciones y las restricciones que presentan las personas con discapacidad generan una carga en la familia, dado el apoyo que deben brindar en los cuidados y las actividades de la vida diaria (Ballesteros et al, 2006; Córdoba et al., 2008).

En las investigaciones citadas anteriormente se observan algunas limitaciones, entre las que se encuentran el tamaño de las muestras utilizadas, ya que en ocasiones no es un número representativo de la población estudiada porque no se cuenta con datos actualizados de la cantidad de personas que presentan las condiciones requeridas, médicas y/o de salud. En las investigaciones que corresponden predominantemente a países europeos, se observa que las muestras de los estudios son más amplias, pero pueden presentar posibles errores en la aplicación de los instrumentos, ya 
que la mayoría de estos son enviados vía correo y no se posee un control directo sobre la forma como las personas los responden.

Adicionalmente, en algunos estudios se observa que el número de ítems incluidos dentro de los instrumentos puede llegar a ser un factor interviniente, ya que en ocasiones los cuestionarios utilizados son largos y generan cansancio, por lo que pueden afectar significativamente los resultados. En general, el diseño de investigación transversal es ampliamente utilizado en los estudios sobre calidad de vida; sin embargo, este diseño impide analizar los efectos a largo plazo de los factores predictores de la calidad de vida. Por tal razón, es importante aumentar la cantidad de investigaciones de corte longitudinal que logren complementar los resultados arrojados por investigaciones de este tipo. Sin embargo, es preciso reconocer que no es fácil desarrollarlas debido a los costos, al acceso restringido a estas poblaciones y a lo inesperado de la condición.

Se han estudiado los factores que generan mayor impacto en la CVRS de las personas. Entre ellos se encuentran las condiciones de salud tales como severidad de los síntomas (Kojima et al., 2009; Krethong et al., 2008; Gaviria et al., 2007; Stuifbergen et al., 2005; Vinaccia et al., 2006a); condición de enfermedad y/o discapacidad adquirida o congénita (Ford et al., 2001; Uppal, 2006); tipo de discapacidad o limitación (Ballesteros et al., 2006); grado de independencia y autonomía en las actividades cotidianas (Pérez et al., 2005). Para las personas que presentan discapacidad física hay una serie de condiciones de salud que influyen sobre su calidad de vida percibida, entre las que se encuentran el tipo de discapacidad (discapacidad de miembro superior, de miembro inferior, dificultades de cadera, ausencias o malformación), el grado de limitación para las actividades cotidianas (entre ellas, si perdió funcionalidad del miembro dominante, si requiere apoyo para traslados y autocuidado entre otras), la ayuda técnica que utilizan (silla de ruedas, muletas, prótesis, bastones, órtesis) y las causas de la discapacidad (enfermedad, violencia social, conflicto armado, accidente de tránsito o laboral), entre otras.

En relación con variables psicológicas se ha encontrado que el afrontamiento es un factor que determina significativamente la calidad de vida en personas con discapacidad o en condiciones crónicas de enfermedad (Abbott et al., 2008; Cano et al., 2006; Contreras et al., 2007; Gaviria et al., 2007; Groot \& Van Den Brink, 2007; Desmond \& MacLachlan, 2006; Taylor et al., 2008; Velasco \& Londoño, 2010; Velasco \& Londoño, 2011; Vinaccia, et al, 2006b). En las personas con discapacidad física, así como en otros grupos poblacionales, pueden existir diferencias importantes en los estilos de afrontamiento utilizados para adaptarse a problemas y dificultades relacionadas con la discapacidad y con la vida diaria. Sin embargo, parece que las personas con discapacidad física tienden a utilizar con mayor frecuencia estrategias de afrontamiento enfocadas en la emoción, especialmente la evitación. Esto se puede relacionar con la forma como los individuos con discapacidad son tratados por su familia y por la sociedad en general, dado un patrón de sobreprotección paternalista y asistencialista que hace que evadan los problemas y tengan dificultad para asumir las consecuencias de sus actos. Sin embargo, es preciso investigar este aspecto ya que la literatura disponible al respecto es limitada.

Otras variables psicológicas asociadas a la calidad de vida de personas con condiciones de enfermedad son los estados emocionales como la ansiedad y la depresión (Albert et al., 2009; Desmond \& MacLachlan, 2006; Endermann \& Zimmermann, 2009; Gallegos et al., 2009; Keeley, et al., 2008; Narváez et al., 2008; Pirkola et al., 2009;); la autoeficacia (Jensen et al., 2009; Pucheu, et al., 2004; Taylor, Dean, \& Siegert, 2006) y la imagen corporal (Carruthers, 2008; Cash, Jakatdar \& Fleming, 2004). Las personas con discapacidad física parecen presentar mayor incidencia de trastornos de ansiedad, trastornos del estado de ánimo y déficit en habilidades sociales e inadecuación social. Adicionalmente, es común encontrar baja percepción de eficacia en personas que han tenido una situación de discapacidad adquirida, por ejemplo en aquellos casos en los que se ha perdido de manera significativa la funcionalidad y la independencia en el ámbito laboral luego de un accidente. La imagen corporal se ve afectada en las personas con discapacidad física, más aun cuando presentan ausencias o malformaciones de miembros superiores y/o inferiores, ya que su imagen corporal se ve distorsionada. Se evita también el reflejo en un espejo que haga evidente la diferencia. Sin embargo, estas observaciones son producto de seguimientos hechos por profesionales sin que se hayan realizado investigaciones sistemáticas al respecto que lo respalden.

En Colombia se han llevado a cabo estudios sobre calidad de vida, predominantemente en la población que presenta enfermedades crónicas como lupus eritematoso sistémico (Vinaccia et al., 2006b), enfermedad pulmonar obstructiva crónica - EPOC (Vinaccia et al., 2006b), cáncer (Gaviria et al., 2007; Vinaccia et al., 2005a), hiperplasia prostática (Vinaccia et al., 2006a), dispepsia no ulcerosa (Cano et al., 2006), artritis reumatoide (Vinaccia et al., 2005b) e insuficiencia renal crónica (Contreras et al., 2007). En relación con la calidad de vida familiar, se han realizado dos investigaciones con familias de personas en situación de discapacidad (Ballesteros, et al, 2006; Córdoba et al., 2008). Esta situación pone en evidencia la importancia de llevar a cabo este estudio en población con discapacidad 
física, dada la ausencia de información proveniente de investigaciones experimentales que vislumbren el estado del arte del tema para esta población.

Dada la situación política de Colombia en cuanto al conflicto armado y el plan del Gobierno de desmovilización, en los últimos años se ha visto un aumento creciente de la población excombatiente y población civil víctima del conflicto armado que, derivado de minas antipersonales (MAP), municiones sin explotar (MUSE) y fuego cruzado, presentan condiciones de discapacidad física que los ha llevado a dar un giro total en las actividades que realizaban antes del evento. Con este panorama, es relevante conocer y determinar el impacto que tienen los factores psicosociales y la situación de discapacidad en la calidad de vida de la población de estudio, generando así un conocimiento que pueda integrarse a los programas y proyectos que van orientados a mejorar la calidad de vida de las personas en situación de discapacidad física a través de talleres y capacitación para que adquieran las habilidades y competencias tendientes a una adecuada inclusión social y laboral en la sociedad.

En suma, la calidad de vida como campo de estudio ampliamente investigado, va a estar determinada por factores relacionados con la situación de discapacidad de las personas, como la severidad de los síntomas, la causa de la discapacidad, el tipo de discapacidad y el grado de autonomía e independencia. Adicionalmente, factores psicosociales como los estilos de afrontamiento, los estados emocionales, la autoeficacia y la imagen corporal pueden afectar la valoración de la calidad de vida de la población con discapacidad. Por ello es importante realizar estudios que permitan conocer más a fondo tanto las variables psicológicas y emocionales de las personas en situación de discapacidad, como sus efectos sobre las condiciones de vida y el bienestar subjetivo.

El presente estudio buscó evaluar los factores psicosociales que determinaban la CVRS en personas con discapacidad física residentes en la ciudad de Bogotá, ya que este conocimiento derivado de la investigación científica puede ayudar a sentar bases claras para las instituciones y entidades no gubernamentales que trabajan para esta población en el marco de la Responsabilidad Social Empresarial en proyectos y programas de inclusión social y laboral.

\section{MÉTODO}

La presente investigación corresponde a un estudio descriptivo correlacional con pretensiones explicativas de predicción entre variables, dada por el tipo de análisis multivariante utilizado (Modelo de Ecuaciones Estructurales).

\section{Participantes}

La muestra de conveniencia estaba conformada por 78 personas entre 18 y 51 años en situación de discapacidad física que hacían parte de un programa de inclusión social y laboral en Bogotá. Entre los participantes, el $41 \%$ eran mujeres y el $59 \%$ hombres, con media de 30 años de edad y desviación estándar de 7.31. La mayoría eran solteros (59\%) o vivían en unión libre (21.8\%); el 42.3\% de las personas tenía secundaria completa, el $24.4 \%$ había realizado algún estudio técnico adicional a la secundaria, y sólo el 6.4\% había realizado estudios universitarios. De acuerdo con la descripción de los participantes en el diseño metodológico, se habían propuesto 100 personas elegidas por conveniencia para la evaluación del modelo predictivo de calidad de vida. Para la evaluación de los factores psicosociales, las condiciones subjetivas y objetivas de la calidad de vida se pudieron evaluar 78 personas con las cuales se corrieron los modelos de ecuaciones estructurales. Se realizó la recolección de la información sobre los factores psicosociales y las condiciones subjetivas de calidad de vida con 101 personas. Sin embargo, no se pudo incluir a 23 participantes dentro de los modelos de ecuaciones estructurales, debido a que por razones de su disponibilidad de tiempo, no fue posible programar la visita domiciliaria para recoger la información correspondiente a calidad de vida objetiva. A pesar de contar con una muestra menor a la esperada, se mantuvo el análisis de ecuaciones estructurales teniendo en cuenta que el límite mínimo admisible por variable, según Casas (2002), es de diez personas.

\section{Instrumentos}

Visita Domiciliaria. Para determinar las condiciones objetivas de $\mathrm{CV}$, se utilizó una ficha de información general para evaluar los diferentes aspectos de las condiciones objetivas de la CV de las personas a través de una visita domiciliaria, tomando como base el instrumento diseñado por Velasco y Londoño (2009) y el instrumento usado en la institución para realizar visitas domiciliarias.

Cuestionario de Salud SF36. La CV se evaluó a través de este instrumento, adaptado y traducido al español por Vilaguta et al. (2005). El SF36 es un instrumento de 36 ítems que evalúa distintas dimensiones: Función física, rol físico, dolor corporal, salud, función social, rol emocional y salud mental. El SF36 evalúa además, la Evolución Declarada de la Salud, a través de la respuesta que la persona da en el ítem 2 , en donde se valora su salud actual en comparación con la salud que tenía hace un año. El instrumento para población hispanoparlante presenta un alfa de Cronbach entre $0.7 \mathrm{y}$ 0.9; los niveles de confiabilidad oscilan alrededor del 0.90 .

Instrumento de Autoeficacia Generalizada EAG. Se utilizó la versión adaptada al español de Baessler y Schwarzer 
(1996 citado por Sanjuán, Pérez, \& Bermúdez, 2000). Este instrumento consta de diez ítems con escala tipo Likert de cinco puntos, que evalúa la autoeficacia general. Esta escala muestra una alta consistencia interna (0.87), con un alfa de Cronbach entre 0.79 y 0.93 , una correlación entre dos mitades de $0.88 \mathrm{y}$ un alto grado de validez convergente $\mathrm{y}$ discriminante.

Inventario de Depresión de Beck BDI. Es un inventario de 21 ítems que evalúan la gravedad (intensidad sintomática) de la depresión en una escala de 0 a 3. Para este estudio se utilizó la versión de Vázquez y Sanz (1991) adaptada y traducida al castellano, con una adecuada consistencia interna (Alfa de Cronbach 0.76 - 0.95).

Escala de Ansiedad de Zung. Esta escala consta de 20 ítems, los cuales se puntúan en una escala Likert de 1 a 4. En Colombia no se ha llevado a cabo un proceso de validación cuya cobertura permita generalizar a toda la población (Pareja \& Campo, 2006). Sin embargo, se han realizado diferentes ejercicios que evalúan el comportamiento estadístico de la prueba en diferentes grupos poblacionales, entre los cuales se ha encontrado una consistencia interna adecuada que oscila entre el 0.69 y 0.78 (Ávila, Escobar, \& Chica, 2005; Cogollo, Díaz, \& Campo, 2006; De la Ossa et al., 2009; Pareja \& Campo, 2006).

Inventario de Estilos de Afrontamiento IEA. Este inventario está constituido por 42 ítems que ayudan a identificar el estilo de afrontamiento que utilizan las personas para enfrentar los eventos estresantes, entre los que pueden estar estilos centrados en la solución de problemas (SP), estilos centrados en la búsqueda de soporte social (SS), estilo evitativo (EE), estilo fantasioso (EF) y estilo autoculpable. Este inventario ha sido validado para población colombiana por Archila, García y Londoño (2005), encontrando una confiabilidad mayor a 0.7, según el Alpha de Cronbach.

Cuestionario de optimismo disposicional para adultos. Este instrumento, elaborado por Londoño et al. (2013), busca determinar la tendencia personal hacia el optimismo disposicional. Consta de 20 ítems que representan situaciones cotidianas de las personas con tres opciones de respuesta: optimista, sesgo optimista y pesimista. Se realizó el proceso de validación del instrumento con una muestra total de 200 adultos, alcanzando un nivel de confiabilidad aceptable de 0.75 .

Prueba de imagen corporal PICDF. Se diseñó un instrumento a partir de la propuesta teórica de Taleporos y McCabe (2001). La escala está enfocada hacia la evaluación de la imagen corporal teniendo en cuenta tres factores: atractivo, funcionalidad y satisfacción. Para tal fin, se realizó el proceso de diseño y validación con evaluación por jueces y una prueba piloto previa a este estudio con 13 personas diferentes a la muestra seleccionada. Para determinar las condiciones psicométricas se hicieron 101 aplicaciones obteniendo una consistencia interna de 0.915 .

\section{Procedimiento}

Para llevar a cabo el estudio, se programó una reunión de presentación general con toda la población en la institución; una vez explicados los objetivos y condiciones del estudio se firmó el consentimiento informado. Posterior a ello, en el mes siguiente se programaron las visitas domiciliarias, de tal modo que el participante y la mayoría de las personas con quienes vivía se encontraran presentes. La investigadora principal realizó las visitas domiciliarias. Una vez éstas finalizaron, se programó una reunión para la aplicación grupal de instrumentos, previa explicación de sus características. Posterior a la recolección de los datos, se procedió a sistematizar la información para realizar los análisis estadísticos teniendo en cuenta tres niveles: nivel descriptivo, análisis correlacional de las variables teniendo en cuenta el coeficiente de correlación producto momento de Pearson con el paquete estadístico SPSS versión 17, y el Modelo de Ecuaciones Estructurales, utilizando el aplicativo AMOS del SPSS, versión 17. Finalmente, se programó una sesión con los participantes de la investigación para socializar los resultados y ofrecer atención psicológica a las personas que, por sus condiciones especiales, requerían intervención adicional.

Para los análisis de ecuaciones estructurales se siguen siete pasos consecutivos: a) Desarrollar el modelo con base en la teoría; b) diseñar el diagrama de flujo de relaciones entre variables; c) identificar el nivel de colinealidad de las variables, con el supuesto de partida de que las correlaciones o las covarianzas tienden hacia 0 ; d) correr el modelo, partiendo del supuesto de que los grados de libertad deben tender a $0 ;$ e) evaluar e identificar el modelo, teniendo en cuenta la especificación del modelo de medida; f) determinar el grado de ajuste del modelo en comparación con otros modelos competitivos, eligiendo aquél en que se explique la mayor cantidad de varianza; g) interpretar el modelo a partir de la teoría para analizar si se requieren ajustes de acuerdo con los modelos teóricos de base. Para poder elegir el mejor modelo explicativo se tienen en cuenta los siguientes criterios: varianza explicada mayor al $30 \%(\mathrm{R} 2)$, baja colinealidad entre variables independientes y significancia menor o igual a p 0.05 del Chi Cuadrado calculado. Cabe notar que en ciencias sociales una varianza explicada aceptable está por encima de $30 \%$, debido a la naturaleza compleja de los fenómenos estudiados.

\section{RESULTADOS}

La mayor parte de los participantes tenía discapacidad en miembros inferiores (29.5\%) o en miembros superiores 
(20.5\%). En lo referente a la causa de la discapacidad, en mayor proporción (38.5\%) la causa de la discapacidad eran accidentes generales, y problemas congénitos (24.4\%); cabe notar que el $19.2 \%$ de los casos era debido a violencia social y minas antipersonales o municiones sin explotar. La mayor parte de la población, al momento del estudio, llevaba en promedio 15 años con la discapacidad; el $38.5 \%$ de las personas no usaba ayudas técnicas, y más del $41 \%$ usaba ayudas técnicas como silla de ruedas, muletas, prótesis y bastones canadienses (véase Tabla 1).

El 41\% de las personas reportó padecer dolor crónico de duración entre seis meses y 29 años, principalmente en las extremidades, columna y/o la espalda. Respecto de la CV objetiva, las personas en promedio reportaron condiciones medias de CV en las áreas familiar y educativa; y la $\mathrm{CV}$ subjetiva mostró una puntuación que indica un nivel medio. Se reportan buenas condiciones económicas y un nivel aceptable en las condiciones laborales. En general, los participantes reportan un buen estado salud, con un un nivel moderado de intercambio social; con baja participación en actividades sociales en el tiempo libre y una red de apoyo social pequeña; manifiestan, además, un nivel de trascendencia y religiosidad moderado.

En la CV objetiva total, los resultados muestran un nivel de calidad de vida alto (Ver Tabla 2) y en la CVRS reportaron puntajes altos en salud mental, función social, vitalidad, función física y salud general. Se encontraron puntajes significativamente bajos en dolor corporal, rol emocional y rol físico; se encontró que la mayoría de las personas reportó que su salud había mejorado en alguna medida en el último año, y solo doce personas indicaron que su salud había empeorado. Adicionalmente, se detectaron 29 casos de depresión y 13 casos con ansiedad moderada o severa. Se encontró que sólo dos personas obtuvieron puntajes altos en autoeficacia y la mayoría de los participantes reportó un nivel medio o alto de religiosidad y espiritualidad.

En la imagen corporal se observaron bajos puntajes en el Factor Atractivo, aun cuando la media global indica que hay una buena evaluación de la imagen corporal en personas con discapacidad física. Respecto a los cinco estilos de afrontamiento, se observaron puntajes elevados en solución de problemas, evitación y fantasía, mientras que algunas personas mostraron puntajes más bajos en soporte social y autoculpa. En el Cuestionario de Optimismo Disposicional, se observó una tendencia marcada en las personas hacia un optimismo elevado.

La varianza total explicada para CV fue de $39 \%$, siendo esto aceptable en estudios de ciencias sociales. En orden jerárquico, las variables que más peso de varianza aportaron al modelo son: condiciones de enfermedad (38\%), depresión (34\%), estilo de afrontamiento fantasioso (24\%), religión y espiritualidad (11\%), pesimismo (5\%), ansiedad

Tabla 1

Resultados de las variables asociadas a la situación de discapacidad de los participantes

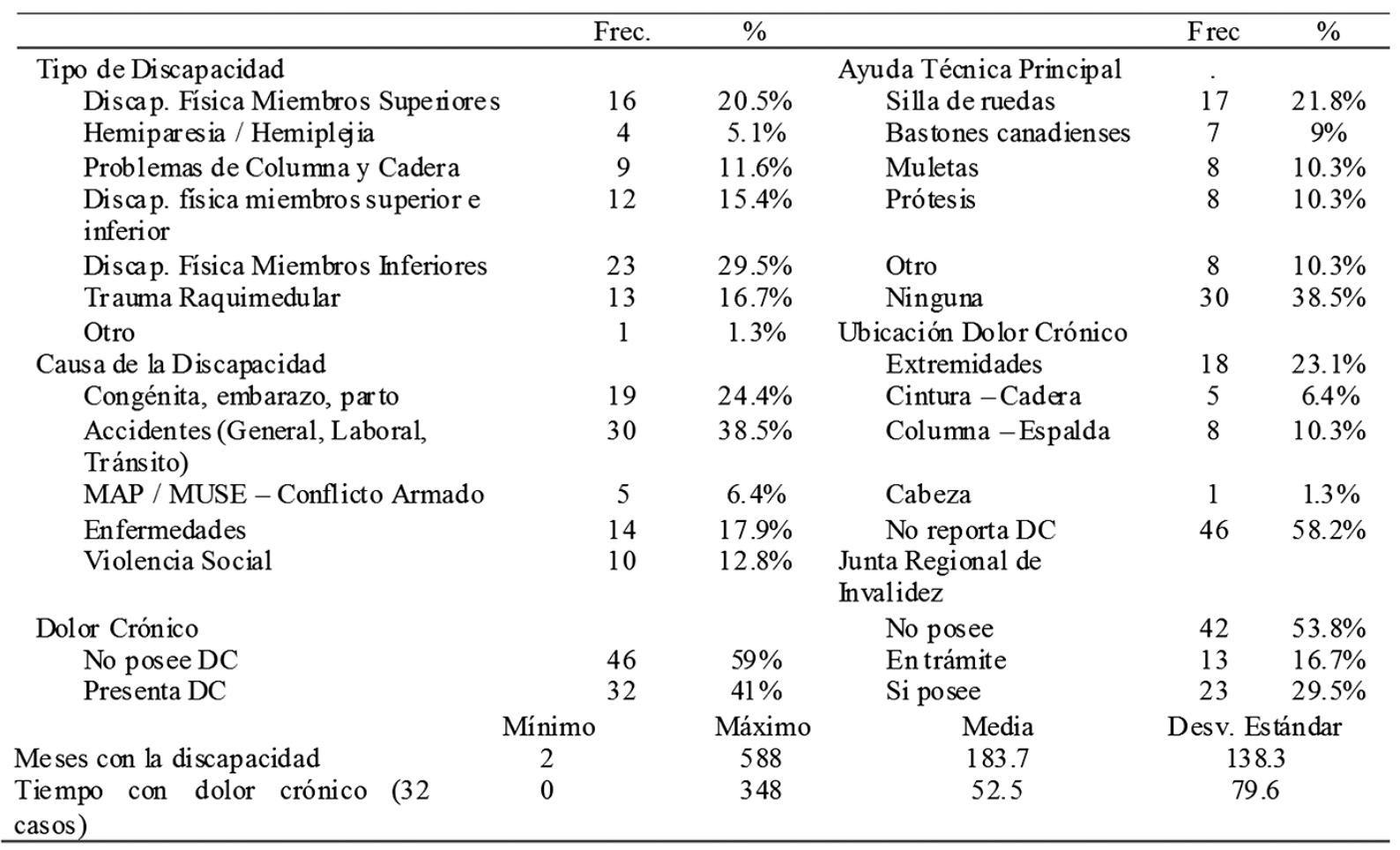


Tabla 2

Resultados de las escalas e instrumentos utilizados

\begin{tabular}{|c|c|c|c|c|c|c|c|c|c|}
\hline & Min. & Max. & $\mathbf{X}$ & ds & & Min. & Max. & $\mathbf{X}$ & ds \\
\hline Calidad de Vida Objetiva & & & & & Imagen Corporal & & & & \\
\hline Área Familiar & .00 & 12 & 6.88 & 2.82 & Puntaje Total & 40.69 & 82.84 & 64.84 & 8.74 \\
\hline Área Educativa & .00 & 6 & 2.5 & 1.56 & Atractivo & 34.78 & 81.16 & 60.44 & 9.63 \\
\hline Condiciones Materiales & 9 & 24 & 17.81 & 3.66 & Funcionalidad & 41.27 & 79.37 & 60.85 & 10.45 \\
\hline Condiciones Económicas & .00 & 6 & 5 & 1.58 & Satisfacción & 14.81 & 100 & 73.22 & 16.41 \\
\hline Área Laboral & .00 & 6 & 3.42 & 2.81 & Afrontamiento & & & & \\
\hline Estado de Salud & 6 & 18 & 14.15 & 3.95 & Autoculpa & .00 & 12 & 7.99 & 2.26 \\
\hline Área Social & .00 & 12 & 6.88 & 2.82 & Sol. problemas & .00 & 40 & 23.01 & 8.01 \\
\hline Trasc, Y Religiosidad & .00 & 15 & 7.65 & 3.41 & Soporte Social & .00 & 17 & 8.76 & 3.71 \\
\hline Puntaje Total & 39 & 84 & 64.31 & 10.45 & Evitación & 6 & 23 & 17.38 & 3.39 \\
\hline Calidad de Vida Subjetiva & & & & & Fantasía & .00 & 24 & 13.21 & 5.14 \\
\hline Función Física & 5 & 100 & 59.94 & 26.30 & Centrado Emoción & 18 & 55 & 40.69 & 8.27 \\
\hline Rol Físico & .00 & 25 & 12.58 & 26.30 & Centrado Problema & 6 & 51 & 29.65 & 9.76 \\
\hline Dolor Corporal & .00 & 100 & 48.83 & 31.80 & Opt.Disposicional & 5 & 20 & 15.56 & 2.84 \\
\hline Salud General & 40 & 87 & 59.76 & 8.76 & Sesgo Optimista & .00 & 7 & 2.97 & 1.57 \\
\hline Vitalidad & 6.25 & 100 & 68.91 & 18.35 & Pesimismo & .00 & 10 & 1.47 & 1.98 \\
\hline Función Social & 25 & 100 & 80.61 & 19.6 & Depresión & .00 & 42 & 8.40 & 7.39 \\
\hline Rol Emocional & .00 & 25 & 15.17 & 10.72 & Ansiedad & 25 & 71 & 38.04 & 7.62 \\
\hline \multirow[t]{2}{*}{ Salud Mental } & 15 & 100 & 76.54 & 18.20 & Autoeficacia General & 11 & 50 & 39.32 & 7.63 \\
\hline & & & & & Relig/espiritualidad & 1 & 28 & 18.64 & 6.71 \\
\hline
\end{tabular}

(4\%) y autoeficacia (3\%). El Chi cuadrado calculado para este modelo fue de 50, con una significancia de 0.6 y 29 grados de libertad. Se eligió este modelo explicativo dado que era el mejor modelo posible, con la mayor varianza explicada $\left(\mathrm{R}^{2}\right)$. Variables como Optimismo, Sesgo Optimista, Variables en relación con la discapacidad, Imagen Corporal y variables sociodemográficas no se incluyeron dentro del modelo debido al bajo aporte a la varianza total de la CV. Otras variables tales como pesimismo, evitación y autoculpa se eliminaron del modelo por la alta colinealidad que presentaron con variables como depresión y ansiedad (véase Figura 1).

\section{DISCUSIÓN}

El interés por la CVRS en parte se deriva del desarrollo tecnológico que ha dispuesto estrategias que permiten extender la vida y mejorar las condiciones de vida de personas con enfermedades crónicas y degenerativas, alineado con ajustes no sólo en las políticas de gestión y de disponibilidad de recursos, sino también en las decisiones clínicas (Costa, Neto, \& Cavalcanti, 2005). En relación con la CVRS, es interesante observar que las personas con discapacidad física incluidas en este estudio, en comparación con otros grupos poblacionales, parecen no tener diferencias con quienes padecen enfermedades crónicas, e incluso tienen

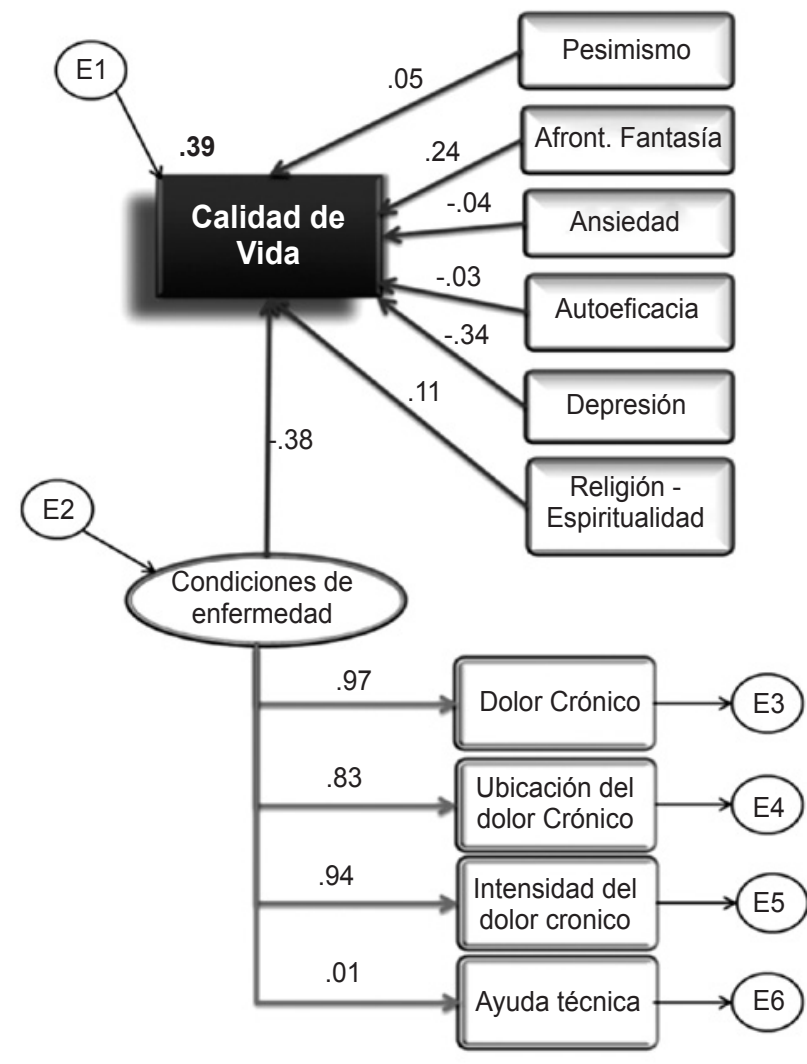

Figura 2. Modelo predictivo de Calidad de Vida. 
mejor CVRS que éstas en esferas como rol social y función física, pero menor puntaje en los roles físico y emocional, y la experimentación de dolor crónico.

Esta comparación es posible dado que el instrumento (SF-36) ha sido ampliamente usado a nivel internacional y con diferentes grupos poblacionales (Vilaguta et al., 2005). La razón de que los participantes hayan obtenido puntajes comparativamente menores a los del resto de la población en la escala de rol físico, puede ser debido al tipo de discapacidad física y la severidad de la misma, ya que aunque estas personas no reportan limitaciones en las actividades cotidianas (función física), sí refieren interferencia significativa en la participación de espacios educativos, laborales y sociales, debidas a su discapacidad (rol físico). Así mismo, refieren interferencia de los problemas emocionales en diversas esferas de su vida, en parte explicada por los problemas de depresión y ansiedad reportados. El dolor crónico también es un indicador de baja CVRS percibida, por el efecto sobre la vivencia de bienestar y satisfacción en las áreas social, laboral y educativa.

En esta misma línea, la discapacidad física es una condición transversal que afecta todas las esferas y componentes de la CVRS de las personas evaluadas; de la misma manera que las enfermedades crónicas, tiene un carácter permanente e irreversible y está asociado con muchos problemas de adaptación. Así como se observa en las enfermedades crónicas, en la discapacidad física la CVRS va a estar determinada por la experiencia del enfrentamiento de las limitaciones cotidianas que produce la discapacidad, junto con los síntomas somáticos como el dolor crónico asociado, las reacciones emocionales que se producen y la percepción de control que pueden tener en cuanto al manejo de la situación de discapacidad (Joyce Moniz \& Barros, 2007).

En comparación con poblaciones que reportan enfermedades crónicas, las personas con discapacidad física de este estudio presentan mejor satisfacción y bienestar psicológico en la esfera de rol social, salud mental y vitalidad, factores que redundan en una mejor CV. Esto debido a que la severidad de las limitaciones físicas que presentan y el grado de funcionalidad y autonomía en la realización de actividades cotidianas, facilitan la satisfacción general y la CV percibida acerca de la vitalidad, el rol social ejercido y la salud mental. Así mismo, es importante notar que estas personas hacen parte de un proyecto de inclusión laboral, y durante los intercambios sociales formales e informales comparten valores, intereses y reciben apoyo emocional que les facilita el manejo de situaciones estresantes (Birditt \& Antonucci, 2008; Moser, 2009). No obstante, cabe resaltar que el hecho de que las personas incluidas en el estudio provengan de una institución que realiza un proceso de selección para el ingreso en el que se evalúa la salud mental (grado de ajuste psicológico y bienestar en la esfera emocional y conductual), sesga los datos obtenidos y por tanto deben conducirse estudios posteriores en población no seleccionada previamente.

En cuanto a la autoeficacia general, se observaron puntajes elevados, hallazgo similar al de estudios previos en jóvenes con discapacidad física (Bent et al., 2001), que indican alta expectativa de obtener los resultados y la percepción de poseer la capacidad personal para alcanzar dicho resultado (Gómez et al., 2007), ambas características relacionadas con la percepción de control que se tiene sobre la enfermedad (Taylor et al., 2006). Las atribuciones que realizan las personas con discapacidad física sobre el logro o los resultados obtenidos en diversas esferas de su vida van a determinar el esfuerzo futuro por resolver la situación, dependiendo del locus de control, la estabilidad y la controlabilidad de la situación (Flórez, 2007). Adicionalmente, la posibilidad de recibir un ingreso económico estable que facilite el mejoramiento de las condiciones de CV y el ajuste a la discapacidad también ha sido relacionada en estudios previos en pacientes con artritis reumatoide y con personas en situación de discapacidad (Vinaccia et al., 2005), ya que las personas incrementan las opciones de elección, elevando el sentido de competencia y confianza (autoeficacia).

Las personas en situación de discapacidad física utilizan con mayor frecuencia estrategias centradas en la emoción, principalmente la evitación. Este hallazgo se asocia con el grado de incontrolabilidad y de permanencia de la situación de discapacidad que hace que en este contexto las estrategias orientadas a solucionar el problema no resulten tan efectivas, tal como lo habían planteado previamente Abbott et al. (2008) y como se observa en pacientes con cáncer (Gaviria et al., 2007) y en mujeres con artritis reumatoide (Carlisle et al., 2005). La adaptación, en casos de enfermedad crónica o discapacidad, va a implicar un ajuste entre las demandas y expectativas que exige un evento que causa una limitación permanente y las capacidades de las personas para dar respuesta a estas demandas; pero como en estos casos no hay posibilidades de cambiar la situación, las estrategias orientadas a la emoción ayudan a reducir el impacto emocional que genera el evento, y a largo plazo, a lograr un equilibrio ante la situación (Rodríguez, Pastor, \& López, 1993). Los resultados encontrados en esta investigación son contrarios a los reportados por Desmond y MacLachlan (2006) en personas con amputaciones, y por Contreras et al. (2007) en pacientes con insuficiencia renal crónica, en donde la evitación se asocia con un ajuste psicológico mucho más pobre y una mayor frecuencia en el uso de estrategias centradas en el problema. 
De la misma manera que los pacientes con lupus eritematoso evaluados por Vinaccia et al. (2006c), las personas en situación de discapacidad física mostraron una tendencia marcada hacia el optimismo disposicional en comparación con el pesimismo y el sesgo optimista, a pesar de las limitaciones y restricciones derivadas de la discapacidad. Esta tendencia al optimismo determina la capacidad de las personas con discapacidad de incluirse activamente en espacios sociales y continuar con sus actividades habituales a pesar de las limitaciones que genera la discapacidad. El optimismo disposicional, entendido como las expectativas o creencias estables y generalizadas positivas acerca del futuro, se relaciona con proyectos de vida realizables y sostenibles promovidos por Eccos, y en circunstancias donde se evalúan pocas oportunidades de cambiar la situación, como en el caso de la discapacidad, las estrategias centradas en la emoción van a resultar de mucha utilidad conjugadas con expectativas optimistas que favorecen la adaptación (Ortíz, Ramos, \& Vera-Villarroel, 2003).

De otra parte, se observa que la mayor parte de las personas incluidas en este estudio refieren una valoración positiva de su imagen corporal, especialmente en la esfera de la satisfacción con el cuerpo. Estar en una situación de discapacidad física puede generar un impacto en la imagen corporal de acuerdo con los estándares de belleza y los ideales del cuerpo establecidos socialmente, en los que la discapacidad hace que haya disonancia al hacer comparaciones con dichos estándares. Los resultados hallados en este estudio no son extrapolables a todas las personas con discapacidad física, dado que el grupo de participantes forma parte de una entidad sin ánimo de lucro que ofrece la inclusión social y laboral, y por ello el soporte social y la posibilidad de referenciarse a sí mismos con personas que tienen la misma condición hace que la aceptación a la discapacidad y la confianza en sí mismos mejoren significativamente $\mathrm{y}$, por ende, aumente la valoración de su imagen corporal (Cash et al., 2004). Así mismo, la adecuada valoración de la imagen corporal que reportaron las personas del estudio se relaciona con la severidad de la discapacidad, el grado de la limitación y la dependencia de cuidadores para las actividades cotidianas, lo cual además facilita su participación en contextos laborales y sociales (Taleporos \& McCabe, 2005).

\section{Predictores de la calidad de vida en personas con discapacidad fisica}

De acuerdo con el modelo hallado, los predictores de $\mathrm{CV}$ de las personas con discapacidad son en orden de peso predictivo: las condiciones de la enfermedad (dolor crónico, su ubicación e intensidad, y el uso de ayudas técnicas); la depresión, el afrontamiento centrado en la fantasía, la trascendencia (religión/espiritualidad); el pesimismo; la ansiedad y la autoeficacia, que en interacción explican el $39 \%$ de la varianza. Cabe anotar que el tamaño de la muestra del estudio corresponde al mínimo requerido para este tipo de análisis, y que sería deseable contrastar los resultados con nuevos estudios, sin que esto reste importancia a los hallazgos aquí reportados. La experiencia dolorosa merma los recursos psicológicos de las personas, ya que éstas experimentan una disminución física y corporal que las hace sentirse menos valiosas que otras personas. Dicha experiencia se ve agudizada por factores como: a) la intensidad del dolor y la ubicación de éste, en términos de la manera en que afecta la funcionalidad de la persona; $b$ ) el uso de ayudas técnicas, que en ocasiones incrementa el dolor o el grado de limitación en las actividades de la vida cotidiana y restringen la participación social. Esto se suma al hecho de presentar síntomas de depresión y ansiedad, probablemente originados por la discriminación social, laboral y educativa a la que han estado expuestos, y que se mantienen por la tendencia pesimista típica de personas que han vivido situaciones trágicas o eventos traumáticos, como en el caso de la discapacidad. Sin embargo, esta tendencia pesimista hace que las expectativas y las metas a alcanzar que se propongan las personas sean menos ambiciosas y que requieran menos, en términos de condiciones de vida, para sentirse satisfechas.

De otra parte, la alta autoeficacia facilita la percepción de bienestar, ya que el individuo percibe como alcanzables las metas propuestas y cree que su comportamiento va a producir los resultados esperados, lo cual está apoyado en las creencias religiosas y espirituales que le dan la sensación de tener soporte social y le otorgan sentido a su proyecto de vida. En las personas con discapacidad física, el afrontamiento centrado en la emoción, específicamente en la fantasía, provee un recurso psicológico para responder ante una situación que no tiene modificación; el individuo recurre a fantasías a través de las cuales logra el ajuste óptimo y en las que probablemente la discapacidad está ausente. No obstante, este hallazgo requiere de mayor investigación para ser comprendido plenamente. Finalmente, los resultados encontrados conducen al rechazo de la hipótesis de trabajo formulada, ya que variables como la imagen corporal y algunas variables sociodemográficas no fueron incluidas en el modelo.

La imagen corporal y su fuerte relación con los niveles depresión y seguridad en sí mismo son variables que influyen en la $\mathrm{CV}$, especialmente en la dimensión subjetiva de bienestar psicológico (Cummins, 2005; Taleporos \& McCabe, 2002). De acuerdo con el marco teórico de referencia, y con estudios como el de Da Rocha et al. (2009), el 
de Boelhouwer y Roes (2004), se reconoce la interferencia que produce la ansiedad y la depresión en los procesos de adaptación y ajuste a la discapacidad física y en la CVRS. En las personas evaluadas se observaron puntajes elevados en ansiedad y depresión. Estos resultados se ajustan a estudios previos que demuestran la fuerte influencia y relación que existe entre los estados emocionales y afectivos y la $\mathrm{CV}$ de las personas que sufren condiciones crónicas de salud, en los que se observa que la depresión es un factor de riesgo para el aumento de las limitaciones físicas generadas por una enfermedad crónica (Ayatollahi et al., 2007; Blane, Netuveli, \& Montgomery, 2008; Jiang et al., 2004;). Dada esta influencia, se sugiere el diseño de programas de prevención de ansiedad y depresión que permitan entrenar a las personas con discapacidad física en técnicas que les ayuden a mejorar su CV, teniendo una adecuada regulación emocional.

Acorde con las propuestas de Diener y Suh, (1997), de Gómez et al. (2007) y de Lieberman y Winzelberg (2009), en el presente estudio no se encontró asociación entre las dimensiones subjetivas y objetivas de la $\mathrm{CV}$, ya que la valoración subjetiva del bienestar está determinada en parte por las condiciones materiales y objetivas, pero en mayor medida por variables psicológicas como la depresión, los estilos de afrontamiento centrados en la emoción como la fantasía, la trascendencia (religión y espiritualidad), el pesimismo, la ansiedad y la autoeficacia (López, Fernández, \& Polo, 2005). En personas con discapacidad física, la valoración subjetiva que éstas hacen sobre sus condiciones de vida en todas las esferas, y la interacción entre sus recursos, competencias y estado funcional del cuerpo con las condiciones que le ofrece el contexto familiar, social y comunitario, van a determinar el ajuste y el bienestar percibido (Liberalesso, 2002). Los resultados del presente estudio favorecen la idea de que las oportunidades que ofrece el medio de integración y participación para las personas con discapacidad son importantes en la valoración de la CV, pero no son suficientes teniendo en cuenta las expectativas de la persona, su proyecto de vida, la experiencia privada y subjetiva de cada persona y los estados emocionales y afectivos que van a mediar la interacción de la persona en condición de discapacidad con su mundo.

En general, las iniciativas gubernamentales y no gubernamentales deben tener en cuenta que la inclusión social de personas en situación de discapacidad involucra necesariamente, tanto las habilidades del individuo para desenvolverse socialmente, como la disposición de la sociedad en general para aceptar a las personas con las diferencias y limitaciones propias de su condición. Las personas en situación de discapacidad tienen barreras inter- nas que dificultan su participación en actividades sociales, recreativas, educativas y laborales, en relación con las limitaciones funcionales, fatiga, pasividad, retraimiento, depresión, vulnerabilidad al estrés, entre otras. No obstante, hay barreras externas relacionadas con el medio físico y social donde se desenvuelven las personas, las cuales pueden ser controladas a través de iniciativas y programas para la población. Entre estas barreras se encuentran los obstáculos para la movilidad, los obstáculos urbanísticos, de transporte, las actitudes sociales estereotipadas, la falta de oportunidades y la dificultad de acceso a los recursos (Ibañez \& Mudarra, 2004).

Dada la alta incidencia de la situación de discapacidad en la población colombiana y el interés creciente del Gobierno, el sector privado y las comunidades en programas de inclusión eficaces, se pretende analizar los factores determinantes de la calidad de vida de las personas en situación de discapacidad para, así mismo, ofrecer lineamientos claros para la formulación de programas y proyectos que pretendan beneficiar a dicha población.

\section{Limitaciones y futuras direcciones}

Las limitaciones de esta investigación están relacionadas con el hecho de que todos los participantes provienen de una única institución (muestreo por conveniencia) y están incluidos en un programa de apoyo, hechos que pueden afectar los resultados. Otra dificultad para la generalización de los resultados se relaciona con la diversidad encontrada en los tipos de discapacidades de la muestra y las diferencias que se observan en el proceso de ajuste y adaptación a la discapacidad de personas con amputaciones, trauma raquimedular, enfermedades crónicas que producen limitaciones físicas, entre otras. La severidad, el tiempo que llevan con la discapacidad, el tipo de discapacidad y las causas que la producen hacen que el grupo evaluado no sea homogéneo y las conclusiones no sean generalizables a otros grupos poblacionales. Estos factores se constituyen en una invitación para continuar en esta línea de investigación con muestras más grandes y diseños de investigación diferentes que permitan ampliar el cuerpo de conocimientos en el área de la discapacidad en Colombia. No obstante, para la recolección de la información se utilizaron instrumentos validados y un método de recolección de información que permite un panorama completo e integral del fenómeno como lo es la visita domiciliaria, en la que se verificaron las condiciones objetivas de $\mathrm{CV}$, y se pudo contrastar información con fuentes alternas. Así mismo, otro de los aportes significativos del presente estudio radica en la elaboración y validación inicial de una prueba específica de imagen corporal para la población. 


\section{REFERENCIAS}

Abbott, J., Hart, A., Morton, A., Gee, L., \& Conway, S. (2008). Health - related quality of life in adults with cystic fibrosis. Journal of Psychosomatic Research, 64, 149 - 157.

Albert, U., Maina, G., Bogetto, F., Chiarle, A. \& Mataix - Cols, D. (2009). Clinical predictors of health - related quality of life in obsessive - compulsive disorder. Comprehensive Psychiatry, 63, 1003-1009. En: http://www.ncbi.nlm.nih. gov/pmc/articles/PMC2633465/

Anctil, T.M., McCubbin, L.D., O’Brien, K.O., Pecora, P., \& Anderson - Harumi, C.A. (2007). Predictors of adult quality of life for foster care alumni with physical and / or psychiatric disabilities. Child Abuse \& Neglect, 31, $1087-1100$

Archila, D., García, D., \& Londoño, C. (2005). Estrategias de afrontamiento y consumo de cigarrillo en estudiantes de la Universidad Santo Tomás. Experiencias Investigativas 2. Universidad Santo Tomás.

Ávila, J., Escobar, F., \& Chica, H. (2005). Prevalencia de ansiedad y depresión en alcohólicos hospitalizados para desintoxicación en Bogotá. Revista de la Facultad de Medicina. Universidad Nacional de Colombia, 53 (4): 219 - 225.

Awad, A.G., Voruganti, L.N.P., \& Heslegrave, R.J. (1997). A conceptual model of quality of life in schizophrenia: Description and preliminary clinical validation. Quality of Life Research, 6, $21-26$.

Ayatollahi, P., Nafissi, S., Eshraghian, M.R., Kaviani, H., \& Tarazi, A. (2007). Impact of depression and disability on quality of life in Iranian patients with multiple sclerosis. Multiple Sclerosis, 13, 275 - 277.

Ballesteros, B.P., Novoa, M., Muñoz, L., Suárez, F., \& Zarante, I. (2006). Calidad de vida en familias con niños menores de dos años afectados por malformaciones congénitas: Perspectiva del cuidador principal. Universitas Psychologica, 5 (3), $457-473$.

Banco Interamericano de Desarrollo (2007) Datos de Discapacidad: Fuentes más recientes sobre tasas de prevalencia en América Latina y el Caribe (ALC). En: http://www.iadb. org/sds/SOC/site 6215 s.htm

Bent, N., Jones, A., Molloy, I., Chamberlain, M.A., \& Tennant, A. (2001). Factors determining participation in young adults with a physical disability: a pilot study. Clinical Rehabilitation, 15, $552-561$.

Birditt, K., \& Antonucci, T.C. (2008). Life sustaining irritations? Relationship quality and mortality in the context of chronic illness. Social Science \& Medicine, 67, 1291 1299.

Blane, D., Netuveli, G., \& Montgomery, S.M. (2008). Quality of life, health and physiological status and change at older ages. Social Science \& Medicine, 66, 1579 - 1587.

Boelhouwer, J. \& Roes, T. (2004). The Social State of the Netherlands: A Model Based Approach to Describing Living Conditions and Quality of Life. Challenges for the Quality of Life in Contemporary Societies Kluwer Academic Publishers. 1 - 15. En: subversion.assembla.com/svn/ epsc.../GIS/.../Jeroen\%20Boelhouwer.pdf
Cano, E., Quiceno, J., Vinaccia, S., Gaviria, A.M.; Tobón, S., \& Sandín, B. (2006). Calidad de vida y factores psicológicos asociados en pacientes con diagnóstico de dispepsia funcional. Universitas Psychologica, 5 (3), $511-520$.

Caqueo, A. \& Lemos, S. (2008). Calidad de vida y funcionamiento familiar de pacientes con esquizofrenia en una comunidad latinoamericana. Psicothem, 20, (4), 577 - 582.

Carlisle, A.C., John, A.M., Fife - Schaw, C., \& Lloyd, M. (2005). The self-regulatory model in women with rheumatoid arthritis: Relationships between illness representations, coping strategies, and illness outcome. British Journal of Health Psychology, 10, 571 - 587.

Carruthers, G. (2008).Types of body representation and the sense of embodiment. Consciousness and Cognition, 17, 1302 -1316 .

Casas, M. (2002).The Multivariate Analysis. California, USA: Plenum Press.

Cash, T.F., Jakatdar, T.A., \& Fleming, E. (2004). The Body Image Quality of Life Inventory: further validation with college men and women. Body Image, 1, 279 - 287.

Cogollo, Z., Díaz, C., \& Campo, A. (2006). Exploración de la validez de constructo de la escala de Zung para depresión en adolescentes escolarizados. Colombia Médica, 37 (2) $102-106$.

Contreras, F., Esguerra, G.A., Espinosa, J.C., \& Gómez, V. (2007). Estilos de afrontamiento y calidad de vida en pacientes con insuficiencia renal crónica (IRC) en tratamiento de hemodiálisis. Acta Colombiana de Psicología, 10 (2), $169-179$.

Córdoba, L., Gómez, J., \& Verdugo, M.A. (2008). Calidad de vida familiar en personas con discapacidad: un análisis comparativo. Universitas Psychologica, 7 (2), 369 - 383.

Costa Neto, S.B. \& Cavalcanti, T.C. (2005). Calidad de vida de los portadores de neoplasia de cabeza y de cuello en fase diagnóstica. Revista Colombiana de Psicología, 14, 53 - 63.

Cummins, R.A. (2005). Moving from the Quality of Life Concept to a Theory. Journal of Intellectual Disability Research, 40 (10), $699-706$.

DANE (2005) Boletín Censo General 2005. Discapacidad Colombia. En: http://www.dane.gov.co/censo/files/boletines/discapacidad.pdf

Da Rocha, N., Power, M., Bushnell, D., \& Fleck, M. (2009). Is there a measurement overlap between depressive symptoms and quality of life? Comprehensive Psychiatry, 50 (6), 549555.

De la Ossa, S., Martínez, Y., Herazo, E., \& Campo, A. (2009). Estudio de la consistencia interna y estructura factorial de tres versiones de la escala de Zung para ansiedad. Colombia Médica, 40 (1) 71 - 77.

Desmond, D. \& MacLachlan, M. (2006). Coping strategies as predictors of psychosocial adaptation in a sample of elderly veterans with acquired lower limb amputations. Social Science \& Medicine, 62, 208 - 216.

Diener, E. \& Suh, E. (1997). Measuring quality of life: economic, social, and subjective indicators. Social Indicators Research, 40, 189 - 216. 
Endermann, M. \& Zimmermann, F. (2009). Factors associated with health-related quality of life, anxiety and depression among young adults with epilepsy and mild cognitive impairments in short-term residential care. Seizure, 18, 167 175.

Flórez, L. (2007). Psicología Social de la Salud. Promoción y prevención. Bogotá, D.C.: El Manual Moderno Ltda.

Ford, H.L., Gerryoe, E., Johnson, M.H., \& Tennant, A. (2001). Health status and quality of life of people with multiple sclerosis. Disability and Rehabilitation, 23 (12), 516 - 521.

Gallegos, K., García, C., Mudgal, J., Romero, X., Durán, L., \& Salmerón, J. (2009). Role of depressive symptoms and comorbid chronic disease on health-related quality of life among community-dwelling older adults. Journal of Psychosomatic Research, 66, $127-135$.

Gaviria, A.M., Vinaccia, S., Riveros, M.F., \& Quiceno, J.M. (2007). Calidad de vida relacionada con la salud, afrontamiento del estrés y emociones negativas en pacientes con cáncer en tratamiento quimioterapéutico. Psicología desde el Caribe, 20, 50 - 75.

Gómez, V., Villegas, C., Barrera, F., \& Cruz, J.E. (2007). Factores predictores de bienestar subjetivo en una muestra colombiana. Revista Latinoamericana de Psicología, 39 (2), $311-325$.

Groot, W. \& Van Den Brink, H.M. (2007). Optimism, pessimism and the compensating income variation of cardiovascular disease: A two-tiered quality of life stochastic frontier model. Social Science \& Medicine, 65, 1479 - 1489.

Ibañez, P. \& Mudarra, M.J. (2004). Relaciones sociales de personas con discapacidad, en el ocio y tiempo libre. Revista Española de Pedagogía, LXII (229), 521 - 540.

Jensen, I.B., Busch, H., Bodin, L., Hagberg, J., Nygren, A., \& Bergstrom, G. (2009). Cost effectiveness of two rehabilitation programmes for neck and back pain patients: A seven year follow-up. Pain, 142, 202-208.

Jiang, J., Tang, Z., Futatsuka, M., \& Zhang, K. (2004). Exploring the influence of depressive symptoms on physical disability: A cohort study of elderly in Beijing, China. Quality of Life Research, 13, $1337-1346$.

Joyce - Moniz, L. \& Barros, L. (2007). Psicología de la enfermedad para cuidados de la salud. Desarrollo e intervención. Bogotá, D.C.: El Manual Moderno, Ltda.

Keeley, P., Creed, F,; Tomenson, B., Todd, C., Borglin, G., \& Dickens, C. (2008). Psychosocial predictors of health-related quality of life and health service utilisation in people with chronic low back pain. Pain, 135, $142-150$.

Kojima, M., Kojima, T., Ishiguro, N., Oguchi, T., Oba, M., Tsuchiya, H., Sugiura, F., Furukawa, T.A., Suzuki, S., \& Tokudome, S. (2009). Psychosocial factors, disease status, and quality of life in patients with rheumatoid arthritis. Journal of Psychosomatic Research, 67, 425-431.

Krethong, P., Jirapaet, V., Jitpanya, C., \& Sloan, R. (2008). A Causal Model of Health - Related Quality of Life in Thai Patients with Heart - Failure, Journal of Nursing Scholarship, 40 (3), $254-260$.

Liberalesso, A. (2002). Bienestar subjetivo en la vida adulta y en la vejez: Hacia una psicología positiva, en América La- tina. Revista Latinoamericana de Psicología, 34 (1-2) 55 -74 .

Lieberman, M.A. \& Winzelberg, A. (2009). The relationship between religious expression and outcomes in online support groups: A partial replication. Computers in Human Behavior, 25, $690-694$.

Londoño, C., Hernández, L., Alejo, I., \& Pulido, D. (2013). Diseño y Validación de la Escala de Optimismo Disposicional/ Pesimismo EOP, Universitas Psychologica, 12 (1) 139-155.

López, M.D., Fernández, C., \& Polo, M.T. (2005). Relación entre el nivel de estudios y el autoconcepto en adultos con discapacidad motora. Psicología Educativa, 11 (2) 99 - 111.

Moser, G. (2009). Quality of life and sustainability: Toward person-environment congruity. Journal of Environmental Psycholog, $1-7$.

Narváez, J.M., Twamley, E.W., McKibbin, C.L., Heaton, R.K., \& Patterson, T.L. (2008). Subjective and objective quality of life in schizophrenia. Schizophrenia Research, 98, 201 -208 .

Organización Mundial de la Salud OMS (2001) Clasificación Internacional del Funcionamiento, la Discapacidad y la Salud CIF. Editado por el Ministerio de Trabajo y Asuntos Sociales, Secretaría General de Asuntos Sociales, Instituto de Migraciones y Servicios Sociales (IMSERSO). En: http:// www.conadis.salud.gob.mx/descargas/pdf/CIF_OMS_ abreviada.pdf

Organización Mundial de la Salud OMS (2005). Disabled often among the 'poorest of poor'. Bulletin of the World Health Organization (BLT) 83 (4). En: http://www.ncbi.nlm.nih. gov/pmc/articles/PMC2626216/

Ortíz, J., Ramos, N., \& Vera - Villarroel, P. (2003). Optimismo y salud: Estado actual e implicaciones para la psicología clínica y de la salud. Suma Psicológica, 10 (1), 119 - 134.

Pareja, A. \& Campo, A. (2006). Prevalencia de síntomas de ansiedad en pacientes con cefalea primaria. Acta Neurológica Colombiana, 22, 299- 303.

Pérez, M.A., Rodríguez, A.M., Galán, A., \& Blanco, A. (2005). Calidad de vida en la salud: Algunas investigaciones en el ámbito hospitalario. Revista Colombiana de Psicología, 14, $64-72$.

Pirkola, S., Saarni, S., Suvisaari, J., Elovainio, M., Partonen, T., Aalto, A., Honkonen, T., Perälä, J., \& Lönnqvist, J. (2009). General health and quality-of-life measures in active, recent, and comorbid mental disorders: a population-based health 2000 study. Comprehensive Psychiatry, 50, $108-$ 114.

Pucheu, S., Consoli, S.M., D’Auzac, C., Francais, P., \& Issad, B. (2004). Do health causal attributions and coping strategies act as moderators of quality of life in peritoneal dialysis patients? Journal of Psychosomatic Research, 56, 317 -322 .

Rodríguez, J., Pastor, M.A., \& López, S. (1993). Afrontamiento, apoyo social, calidad de vida y enfermedad. Psicothema, $5,349-372$.

Sanjuán, P., Pérez, A.M., \& Bermúdez, J. (2000). Escala de autoeficacia general: datos psicométricos de la adaptación para población española. Psicothema, 12 (2), 509 - 513. 
Schwartzmann, L. (2003). Calidad de vida relacionada con la salud: aspectos conceptuales. Ciencia y Enfermería, IX (2), $9-21$.

Stuifbergen, A.K., Seraphine, A., Harrison, T., \& Adachi, E. (2005). An explanatory model of health promotion and quality of life for persons with post-polio syndrome. Social Science \& Medicine, 60, 383-393.

Taleporos, G. \& McCabe, M.P. (2005). The relationship between the severity and duration of physical disability and body esteem. Psychology and Health, 20 (5) 637 - 650.

Taleporos, G. \& McCabe, M.P. (2002). The Impact of Sexual Esteem, Body Esteem, and Sexual Satisfaction on Psychological Well-being in People with Physical Disability. Sexuality and Disability, 20 (3) $177-183$.

Taleporos, G. \& McCabe, M.P. (2001). The impact of physical disability on body esteem. Sexuality and Disability, 19 (4) $293-308$.

Taylor, J., Smith, P., Babyak, M., Barbour, K., Hoffman, B., Sebring, D., Davis, R., Palmer, S., Keefe, F., Carney, R., Cisk, I., Freedland, K., \& Blumenthal, J. (2008). Coping and quality of life in patients awaiting lung transplantation. Journal of Psychosomatic Research, 65, 71 - 79.

Taylor, W.J., Dean, S.G., \& Siegert, R.J. (2006). Differential association of general and health self-efficacy with disability, health-related quality of life and psychological distress from musculoskeletal pain in a cross-sectional general adult population survey. Pain, 125, $225-232$.

Uppal, S. (2006). Impact of the timing, type and severity of disability on the subjective well-being of individuals with disabilities. Social Science \& Medicine, 63, 525 - 539.

Vázquez, C. \& Sanz, J. (1991). Trastornos depresivos (I): Datos clínicos y modelos teóricos. En A. Belloch \& E. Ibáñez (Eds.), Manual de Psicopatología, Vol. 2, pp. 717-784. Valencia: Promolibro.

Velasco, R.M. \& Londoño, C. (2010). Determinantes psicosociales de la permanencia en el programa de reintegración social en desmovilizados. Revista Iberoamericana de Psicología: Ciencia y Tecnología, 2 (2) 17-32.
Velasco, R.M. \& Londoño, C. (2011). Calidad de vida objetiva, optimismo, variables socio-jurídicas predictivas de la calidad de vida subjetiva en colombianos desmovilizados. Avances en Psicología Latinoamericana, 29 (1) 114-128.

Velasco, M., Botero, P., \& Londoño, C, (2011). Calidad de vida y potencial humano. En: C. Londoño Eds., Avances y Perspectivas en Psicología de la Salud, Colegio Colombiano de Psicólogos.

Vilaguta, G., Ferrera, M., Rajmilb, L., Rebolloc, P., Permanyer - Miraldad, G., Qiuntanae, J.M.,

Santeda, R., Valderasa, J.M., Riberad, A.; Domingo - Salvanya, A., \& Alonso, J. (2005). El Cuestionario de Salud SF-36 Español: una década de experiencia y nuevos desarrollos. Gaceta Sanitaria, 19 (2) 135 - 150.

Vinaccia, S., Quiceno, J.M, Fonseca, P., Fernández, H., \& Tobón, S. (2006a). Calidad de vida relacionada con la salud y su impacto sobre la cognición hacia la enfermedad en pacientes con hiperplasia prostática. Acta Colombiana de Psicología, 9 (2), 47 - 56.

Vinaccia, S., Quiceno, J.M., Zapata, C., Obesso, S., \& Quintero, D.C. (2006b). Calidad de vida relacionada con la salud y emociones negativas en pacientes con diagnóstico de enfermedad pulmonar obstructiva crónica (EPOC). Psicología desde el Caribe, 18, 89 - 108.

Vinaccia, S., Quiceno, J.M., Zapata, C., Abad, L., Pineda, R., \& Anaya, J.M. (2006c). Calidad de vida en pacientes con lupus eritematoso sistémico. Revista Colombiana de Psicología, 15, $57-65$.

Vinaccia, S., Quiceno, J.M., Contreras, F., Zapata, M., Fernández, H., Bedoya, M., \& Tobón, S. (2005a). Calidad de vida, personalidad resistente y apoyo social percibido en pacientes con diagnóstico de cáncer de pulmón. Psicología y Salud, 15, $207-221$.

Vinaccia, S., Contreras, F., Restrepo, L.M., Cadena, J., \& Amaya, J.M. (2005b). Autoeficacia, desesperanza aprendida e incapacidad funcional en pacientes con diagnóstico de artritis reumatoide. International Journal of Clinical and Health Psychology, 5 (1) 129 - 142. 